\title{
Steganographic Data Hiding using DWT and Particle Swarm Optimization
}

\author{
E Divya \\ Department of Electronics and Communication \\ Nehru College of Engineering and Research Centre
}

\author{
P.Rajkumar \\ Department of Electronics and Communication \\ Nehru College of Engineering and Research Centre
}

\begin{abstract}
This paper discusses the steganographic data hiding using the wavelet approach and the optimisation technique. The Discrete Wavelet Transform Using Haar Wavelet gives the excellent peak signal to noise ratio (PSNR) and less computation time. The particle swarm optimisation algorithm (PSO) is also used to hide the data so that the PSNR is improved. The results reveal the DWT using Haar wavelet and PSO algorithm gives excellent PSNR.
\end{abstract}

\section{General Terms}

Particle Swarm Optimisation is an evolutionary algorithm like the genetic algorithm.

\section{Keywords}

PSO, DWT, LSB, DCT, PSNR

\section{INTRODUCTION}

Steganography has been used from the past to the present. In the past in ancient times in Greece they wrote secret messages on wood and wax it after that (wax tablets). As a story says during Greek times they send the spy or slaves with a secret messages, they first clean their shaves and tattoo the messages and once their hair grows the send them to the desired place. During World War they wrote these secret messages in paper using invisible inks.

In1985 with the advent of computers digital steganography entered the world. It includes the concealement of information within computer files. The word steganography comes from the Greek word steganos meaning protected and graphia meaning writing .So it is the art of hiding secret message within a file, image, audio or video. The steganography has the advantage that the secret data is concealed (that is the observer doesn't know the existence of data). Whereas in cryptography we feel the existence of data. Steganography finds applications in many fields like computer forensics, military etc. So there are many techniques existing for this and basically it is divided into two spatial domain technique and transform domain technique. Spatial domain techniques includes the least significant bit method (LSB) which is simplest of all the methods but is more prone to attacks. The second includes the cosine transform approach (like the JSteg algorithm) which is less prone to attacks but has less payload capacity compared to the LSB .So we go for another approach the wavelet which has more payload capacity which is described in detailed in the next sections This paper applies the particle swarm optimization technique to get the better results

\section{LITERATURE REVIEW}

Ratnakirti Roy et.al ${ }^{[1]}$ has made an analysis of the various existing techniques in data hiding and has compared various algorithms in the existing spatial and transform domain techniques. Kennedy and Eberhart ${ }^{[2]}$ proposed the particle swarm optimisation which is similar to the genetic algorithm. Feno Heriniaina Rabevohitra and Jun Sang ${ }^{[3]}$ has used the optimisation algorithm (PSO) for simple LSB substitution based steganography Scheme in DCT domain. Anjali A Shejul and Umesh L. Kulkarni ${ }^{[4]}$ has used the wavelet approach in skin tone to hide data and obtained best results. Sabyasachi Pramanik and Samir K. Bandyopadhyay ${ }^{[5]}$ has used genetic algorithm to obtain best result using the wavelet approach.

\section{STEGANOGRAPHIC SYSTEM}

The basic structure of a steganography consists of a cover image, secret information, stego image. The cover can be an image, audio or video. The secret information can be a text message, image ,audio or video. These base images, texts or audio are referred to as cover and the combination secret information and cover is known as stego.

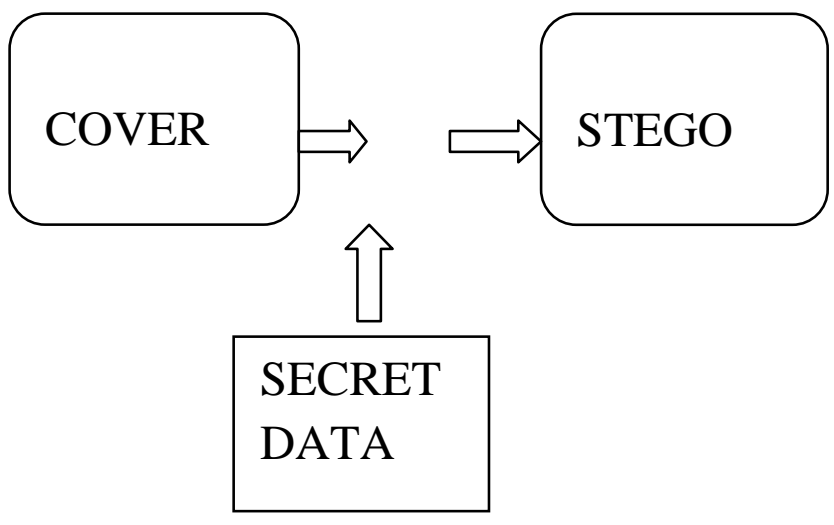

Fig 1: Block Diagram Of Typical Steganographic System

\section{PREVIOUS WORK}

The steganographic data using LSB technique gave excellent performance using the PSO. But the spatial domain technique is more prone to attacks. Instead of LSB technique to improve the security the DCT technique using PSO is proposed but the DCT technique has less payload capacity. The PSO gave excellent performance improvement for both techniques..

\section{WAVELET TRANSFORM}

For the representation of time frequency plots wavelet transform is the most popular. There are many popular types of transform like fourier transform and short fourier transform.But in both cases time and frequency resolutions 
cannot be taken at a time so the wavelet approach is preferred.

\section{1 .Discrete Wavelet Transform}

Discrete Wavelet Transform (DWT) ia any wavelet transform which is discretely sampled which captures both frequency and location information. A wavelet transform splits into basis functions which are known as wavelets. The wavelets are orthogonal, orthonormal, or bi-orthogonal scalar or multiwavelets and convert an carrier into series of wavelet packets than pixel blocks

For an image 2D-DWT is used which decomposes into approximate and detail coefficients(which further into horizontal, vertical and diagonal components).Human visual system are sensitive to the low frequency part the data is hidden in the other three parts without making changes low frequency band. The other three bands are high frequency bands so if there is a small change it is not going to make much change. A 2-D image can be decomposed using a wavelet as shown below

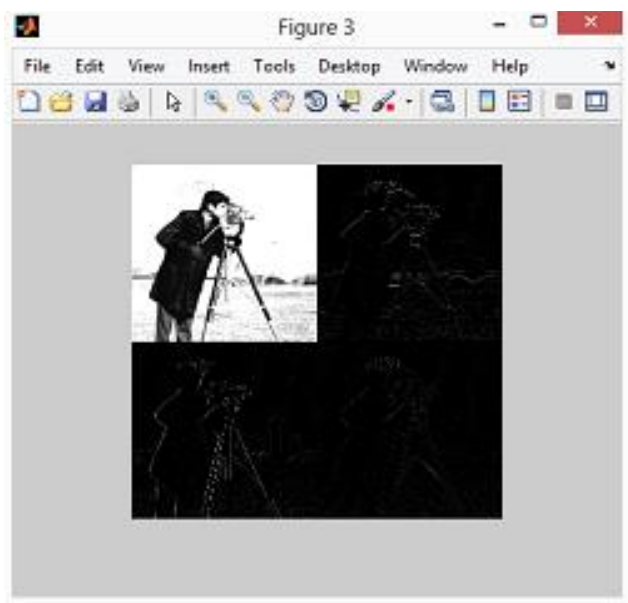

Fig2 : An Image Decomposed Using DWT into 4 bands.

Here Haar wavelet is used .Haar Wavelet is a sequence of rescaled square shaped functions which together form a family or wavelet basis. It was proposed by Alfred Haar in the year 1909.It is the simplest of all wavelet but is not continuous. The Haar wavelet gives the excellent result compared to other wavelet. Haar wavelet is less complex and has less computation time compared to other wavelets.

\subsubsection{Algorithm For Data Hiding Using DWT Technique}

Step 1: Read the cover image and secret message.

Step 2: Convert the input image into bit stream

Step 3: Transform the cover image using DWT

Step 4:Embed the secret data into transformed data

Step 5:Perform the inverse DWT to get the stego image.

Step 6:Write the stego image.

\section{PARTICLE SWARM OPTIMISATION}

Particle Swarm optimisation is an optimisation technique proposed by Kennedy and Eberhart in the year1995.It is an optimisation technique similar to Genetic Algorithms (GAs).The algorithm simulates the behaviour of bird flock flying in search of food in search space. Likewise, the particles fly around the multidimensional space in search of space to find the optimum space to hide the data. Swarms or particles are initialised randomly.

The velocity and position of the particle are initialised randomly. Each particle have to maintain its positions Pbest known as local best position and the Gbest known as global best position among all the particles. That is for each iteration the velocity and position of the particle are updated. Let $\operatorname{Pi}(\mathrm{t})$ denote the position of the particle and this position is changed by adding a velocity component $\mathrm{Vi}(\mathrm{t})$ to it.

$$
P i(t+1)=P i(t)+V i(t+1)
$$

And its velocity is updated by

$$
\begin{gathered}
V i(t+1)=C * W * V i(t)+c 1 * R 1(t) *\left[P_{b e s t}-P i(t)\right] \\
+c 2 * R 2(t) *\left[G_{b e s t}-P i(t)\right]
\end{gathered}
$$

The experiential knowledge of a particle is generally referred to as the cognitive component, which is proportional to the distance of the particle from its own best position found since the first time step. The socially exchanged information is referred to as the social component of the velocity equation. $\mathrm{c} 1$ and $\mathrm{c} 2$ are the cognitive and social components and $\mathrm{c} 1+\mathrm{c} 2$ can be maximum upto 4 . The personal best position Pbest associated with particle $\mathrm{i}$ is the best position the particle has visited since the first time step. $\mathrm{G}_{\text {best }}$ is the global best position for the PSO.R1 and R2 are the two random numbers in the range $[0,1] . \mathrm{C} * \mathrm{~W}$ is the inertia weight introduced to control and balance the exploration and exploitation trade off. $W$ changes according to the number of iteration and the maximum value of $w$ achieved is 0.99 .C is taken to be 1 . The personal best position at the next step can be calculated using:

$\operatorname{Pbest}(t+1)=\operatorname{Pbest}(t)$ if $f(\operatorname{Pi}(t+1)) \geq f(\operatorname{Pi}(t))$

$$
P i(t+1) \text { if } f(P i(t+1))<f(P i(t))
$$

Where $f$ is the fitness function and global best position is calculated as:

$$
\text { Gbest }=\{P 1, P 2, \ldots \ldots \ldots \ldots, P n\}=\min \{f(P 1(t)), \ldots \ldots \ldots f(P n(t))\}
$$

Here the factor $\mathrm{W}$ is calculated by using the equation(5) so that the factor doesnot exceed 0.99

$$
W=(\text { maximum iteration }- \text { iteration }) /(\text { maximum iteration })(5)
$$

\subsubsection{Algorithm For Basic PSO}

Step 1: Initialise the population randomly

Step 2: Start the iteration.

Step 3: Calculate the fitness for each iteration

Step 4:Calculate the local best position and global best position 
Step 5:Update the particle velocity and position using the equation 2 and 3.

\subsubsection{Algorithm For The Proposed Method}

Step 1:Read the cover image and secret message.

Step 2: Convert the input image into bit stream.

Step 3:Intialise the population randomly

Step 4:Transform the cover image using DWT.

Step 5: Embed the secret data into the transformed image

Step 6:Perform the inverse DWT

Step 7: Calculate the fitness for each iteration.

Step 8: Calculate the local and global best position

Step 9:Update the particle velocity and position using the equation 2 and 3

Step 10:Write the stego image

\section{PERFORMANCE MATRICES}

Performance of the above techniques are compared using the Mean square error, Peak signal to noise ratio and computation time.

\subsubsection{Mean Square Error}

The Mean Square Error (MSE) and the Peak Signal to Noise Ratio (PSNR) are the two error metrics used to compare image compression quality. The MSE represents the cumulative squared error between the compressed and the original image.

Mean Square Error $=\operatorname{sum}(\operatorname{sum}($ error $)))^{\wedge} 2 /$ size $($ original image $)$

\subsubsection{Peak Signal To Noise Ratio}

To compute the paek signal to noise ratio(PSNR), the block first calculates the mean-squared error using the following equation. Then the block computes the PSNR using the following equation:

$P S N R=10 * \log _{10}(255 * 255 /$ Mean Square Error $)$

\section{RESULTS AND DISCUSSIONS}

All the results are simulated in matlab software 2010.This paper discusses the performance of the DWT technique using particle swarm optimization. By applying PSO algorithm it is possible to improve the PSNR and decrease the mean square error.This paper comared the results with previous work.The PSO algorithm was carried out for 100 iterations.After 100 iterations the PSNR has improved to a high value. The DWT technique using PSO algorithm gave highest PSNR compared to the DCT and LSB techniques.LSB also gives highest PSNR and has less computation time compared to DWT but since DWT hiding hiding is done in the approximate coefficient it is less prone to attacks.A 335 character is used to hide in test images cameraman,lena and Baboon

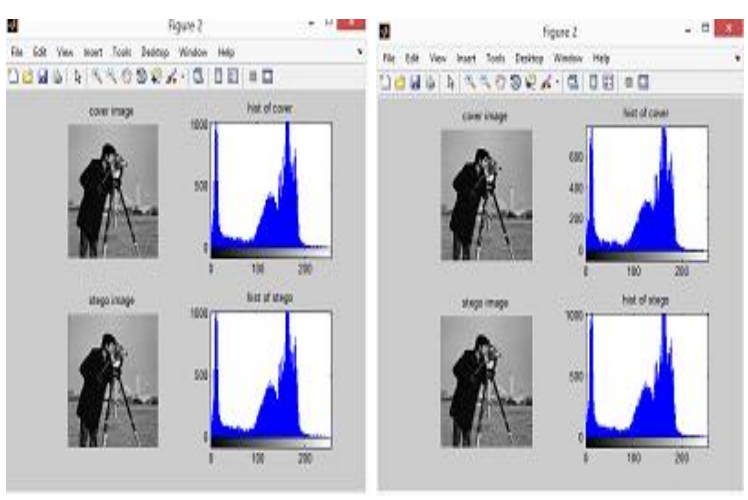

Fig 3: Test Result Of Cameraman Hiding Text Message (a) based on DWT method (right) (b)based on PSO Method (left)

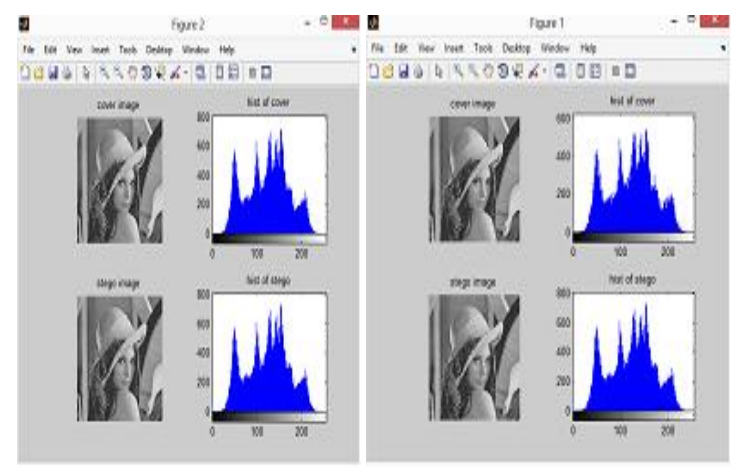

Fig 4: Test Result Of Lena Hiding Text Message (a) based on DWT method (right) (b)based on PSO Method (left)

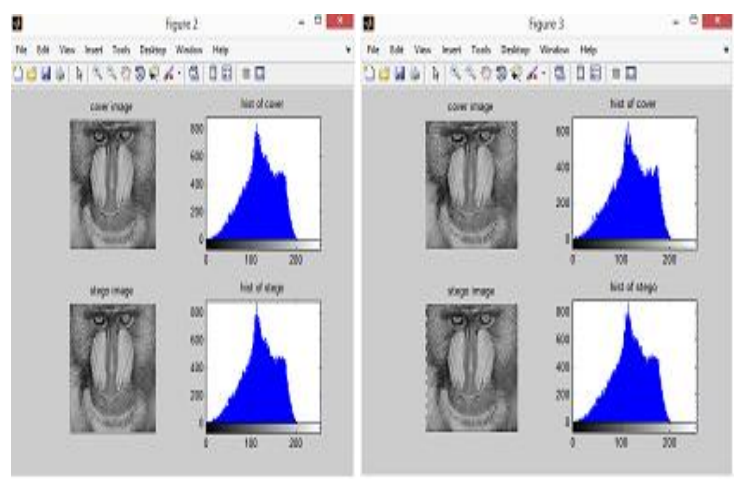

Fig5:Test Result Of Baboon Hiding Text Message (a) based on DWT method (right) (b)based on PSO Method (left)

Table1: PSNR Comparison For Hiding Text Message

\begin{tabular}{|l|l|l|l|l|l|l|}
\hline Image & LSB & $\begin{array}{l}\text { LSB } \\
\text { PSO }\end{array}$ & DCT & $\begin{array}{l}\text { DCT } \\
\text { PSO }\end{array}$ & DWT & $\begin{array}{l}\text { DWT } \\
\text { PSO }\end{array}$ \\
\hline Lena & 50.52 & 70.190 & 28.1 & 68.0 & 32.6 & 72.6 \\
\hline Cameraman & 50.86 & 70.51 & 27.13 & 67.52 & 32.50 & 72.50 \\
& & & & & & \\
\hline Baboon & 50.46 & 70.13 & 32.56 & 49.58 & 38.80 & 78.80 \\
& & & & & & \\
\hline
\end{tabular}


Table2: MSE Comparison For Hiding Text Message

\begin{tabular}{|l|l|l|l|l|l|l|}
\hline Image & LSB & $\begin{array}{l}\text { LSB } \\
\text { PSO }\end{array}$ & DCT & $\begin{array}{l}\text { DCT } \\
\text { PSO }\end{array}$ & DWT & $\begin{array}{l}\text { DWT } \\
\text { PSO }\end{array}$ \\
\hline Lena & 0.580 & 0.006 & 100 & 0.001 & 35.48 & 0.003 \\
\hline Cameraman & 0.537 & 0.005 & 36 & 0.001 & 36.4 & 0.003 \\
\hline Baboon & 0.5886 & 0.0064 & 34.5 & 0.7162 & 8.57 & 0.1557 \\
& & & & & & \\
\hline
\end{tabular}

Table3: Computation Time In seconds

\begin{tabular}{|l|l|l|l|l|l|l|}
\hline $\begin{array}{l}\text { Image(in } \\
\text { seconds) }\end{array}$ & LSB & $\begin{array}{l}\text { LSB } \\
\text { PSO }\end{array}$ & DCT & $\begin{array}{l}\text { DCT } \\
\text { PSO }\end{array}$ & DWT & $\begin{array}{l}\text { DWT } \\
\text { PSO }\end{array}$ \\
\hline Lena & 0.285 & 27.570 & 3.98 & 438.23 & 48.19 & 1846.3 \\
\hline Cameraman & 0.3425 & 38.865 & 3.743 & 461.8 & 49.38 & 1926 \\
\hline Baboon & 0.0739 & 23.8917 & 5.79 & 225.85 & 52.617 & 3681 \\
& & & & & & \\
\hline
\end{tabular}

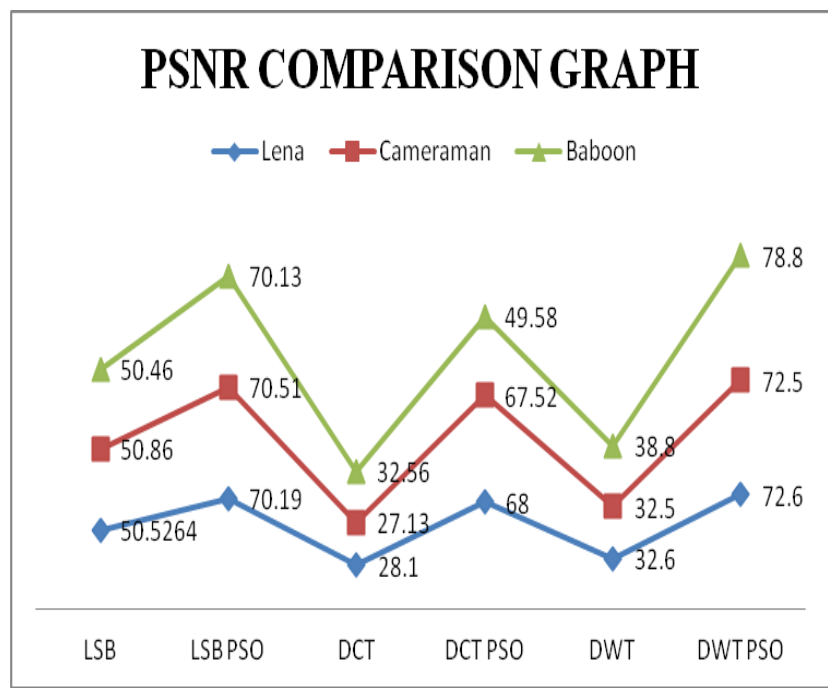

Fig 6:PSNR Comparison Graph

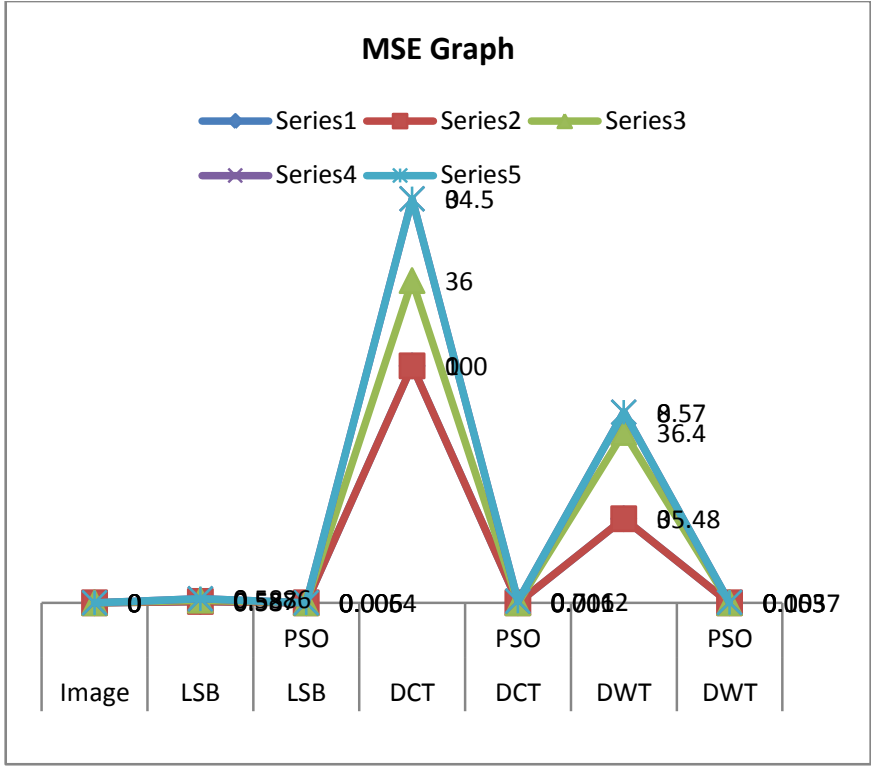

Fig 7:Graphical Comparison of Mean Square Error

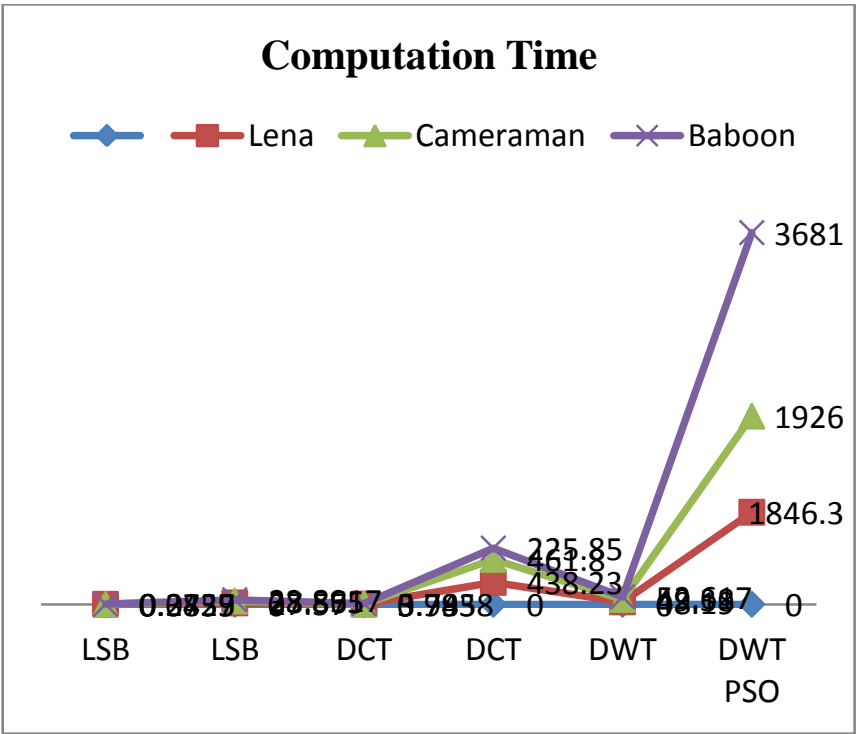

Fig 8:Comparison Of Computation Time(in seconds)

\section{CONCLUSION}

In this paper the basic steganographic Techniques are compared. The DWT technique gives better Performance compared to the DCT and is less prone to attack compared to LSB. In this paper the particle swarm Optimisation is analysed on DWT and it gave better PSNR compared to Others.So the PSO algorithm improves the performance with highest PSNRThe DWT gives excellent payload capacity by applying the particle swarm optimisation technique.The LSB techniques also gives better PSNR but is prone to attack.

The PSO using DWT gives higher PSNR but here the DWT technique takes a higher computation time compared to LSB and DCT.So the future work would be in decreasing the computation time with improved PSNR. 


\section{REFERENCES}

[1] Ratnakirti Roy, Suvamoy Changder1, Anirban Sarkar1, NarayanC Debnath, "Evaluating Image Steganography Techniques: Future Research Challenges" IEEE transactions 2013

[2] Kennedy and Russel Eberhart,"Particle Swarm Optimisation" IEEE International Conference On Neural Networks,Vol6,Nov 1995

[3] Feno Heriniaina Rabevohitra and Jun Sang "Using PSO Algorithm for simple LSB Substitution Based Steganography Scheme in DCT Transformation Domain”@Springer-Verlag Berlin Heidelberg 2011,vol pp.212-220,2011

[4] Anjali A. Shejul, Umesh L. Kulkarni," A Secure Skin Tonebased Steganography Using Wavelet Transform"International Journal Of computer theory and engineering,Vol 3, No.1, pp. 16-22,February,2011

[5] Sabyasachi Pramanik and Samir K. Bandyopadhyay," Image Steganography Using Wavelet Transform And Genetic Algorithm" International Journal Of innovative research in advanced engineering,Vol 1,March 2014

[6] P.Rajkumar, R.Kar, A.K.Bhattacharjee, H.Dharmasa "A comparative analysis of steganographic data hiding within digital Images" International Journal Of Computer Applications, Vol 53. N0-1, September 2012

[7] Emad Elbeltagi, Tarek Hegazy, Donald Grierson, "Comparison among five evolutionary-based optimization algorithms", @ science direct Advanced Engineering Informatics 19 (2005) 43-53

[8] Santi P. Maity and Malay K. Kundu, "Genetic algorithms for optimality of data hiding in digital images" Published online: 31 May 2008@ Springer-Verlag 2008
[9] Haar wavelet - Wikipedia http://en.wikipedia.org/wiki/Haar_wavelet

[10] Wavelet transform - Wikipedia http://en.wikipedia.org/wiki/Wavelet_transform

[11] N. Lavanya, V.Manjula, N.V. Krishna Rao,” Robust and Secure Data Hiding in Image UsingBiometric Technique, (IJCSIT) International Journal of Computer Science and Information Technologies, Vol. 3 (5), $2012,5133-51$.

[12] Rafael C.Gonzalez, Richard E. Woods, Steven L.Eddins, "Digital Image Processing Using MATLAB' Pearson Education, 2004.

[13] Bin Li, Junhui He, Jiwu Huang, Yun Qing Shi, "A Survey on ImageSteganography and Steganalysis", Journal of Information Hiding and Multimedia Signal Processing, Vol.2, Issue 2, pp. 142-172, April 201

[14] C. Cachin, "An information-theoretic model for steganography," Proc.2nd International Workshop Information Hiding LNCS 1525, pp. 306-318, 1998

[15] Muhammad Imran ,et.al,"An Overview Of Particle Swarm Optimisation Variants"Published by Elsevier $1877-7058,2013$

[16] Mohd Afizi Mohd Shukran,et.al,'Image Classification Technique using modified Particle Swarm Optimisation" Modern Applied Science, Vol. 5, No. 5; October 2011

[17] Punam Bedi, Roli Bansal, Priti Sehgal," Using PSO in a spatial domain based image hiding scheme with distortion tolerance",@ science direct Computers and Electrical Engineering 39 (2013) 640-654. 